\title{
Monitorización de la cooperación alcanzada y estimación del rendimiento en trabajos en equipo
}

\author{
Francisco J. Suárez, Víctor Corcoba, María J. Suárez-Cabal, Pablo J. Tuya \\ Universidad de Oviedo, Departamento de Informática, Gijón, España, \\ \{fjsuarez, corcobavictor, cabal, tuya\}@uniovi.es
}

\begin{abstract}
Resumen
En este trabajo se analiza la relación entre el nivel de cooperación alcanzado en equipos y el rendimiento obtenido por los mismos durante el desarrollo de trabajos en grupo en varias asignaturas. El objetivo último es la monitorización en tiempo real del nivel de cooperación alcanzada por los equipos de alumnos con objeto de corregir a tiempo posibles problemas de funcionamiento interno de los mismos. Se busca además familiarizar al alumno con herramientas y estrategias para trabajo cooperativo que mиy probablemente van a encontrar en su futuro laboral.
\end{abstract}

Keywords: innovación educativa, trabajo cooperativo, herramientas TIC.

\section{Introducción}

La iniciativa de innovación que aquí se describe surge como respuesta a la falta de información sobre el funcionamiento interno de los equipos de alumnos que abordan el desarrollo de trabajos en grupo en las asignaturas de grado y máster. Si bien siempre resulta interesante conocer dicha información, en asignaturas donde el trabajo en grupo es la principal actividad evaluada supone una información esencial. Se trata pues de medir de forma objetiva el grado de cooperación alcanzada en los equipos como complemento a la valoración subjetiva de los profesores. En las asignaturas que se han planteado como objetivo resulta además especialmente importante valorar la competencia de capacidad de cooperación del alumno.

La duración de las actividades colaborativas a evaluar es un aspecto de suma importancia, ya que condiciona en gran medida las interacciones entre los estudiantes (Viswanathan, 2017). Gran parte de los estudios que podemos encontrar en la literatura corresponden a actividades colaborativas en el ámbito de una sesión docente, es decir, en torno a una hora. 
En todo caso, existen también algunos estudios sobre equipos de estudiantes que trabajan juntos durante varias semanas o un semestre completo utilizando herramientas tales como foros, wikis, Chat o repositorios de código. Este tipo de trabajos es sin duda mucho más acorde al planteado en nuestro trabajo, ya que vamos a considerar trabajos en equipo cuya duración es de 12 semanas. En el informe Horizon sobre Educación Superior (INTEF, 2017) se proporcionan algunas de estas alternativas para el aprendizaje colaborativo, como son los wikis, Google Docs, las redes sociales y las aplicaciones de mensajería. En Perera (2009) se utilizan métricas de uso de wikis y repositorios de código en el contexto de un proyecto sobre programación desarrollado en equipos de entre 5 y 7 alumnos durante 12 semanas. En Anaya (2011) se utilizan métricas de uso de foros en el contexto de una tarea colaborativa de 12 semanas desarrollada en equipos de 3 alumnos pertenecientes a la Universidad Nacional de Educación a Distancia. En ambos casos, el nivel de colaboración alcanzado tanto por los equipos como por cada uno de sus miembros se valora por parte de expertos y después se intenta inferir dicho nivel a partir de las métricas mediante diferentes técnicas como clustering, data mining y machine learning. También el uso de las redes sociales, junto con la potencialidad ofrecida por herramientas TIC colaborativas como Slack o Teams, pueden fomentar la cooperación entre alumnos y permitir una mayor participación, fomentando el aprendizaje cooperativo (Chawinga, 2017).

\section{Objetivos del estudio}

1. Familiarizar al alumno con herramientas y estrategias de trabajo cooperativo con las que se van a encontrar en su futuro laboral.

2. Medir y monitorizar el nivel de cooperación alcanzado por los alumnos durante el desarrollo de los trabajos en equipo de las asignaturas.

3. Estimar el rendimiento obtenido por los equipos a partir del grado de cooperación alcanzado en los mismos.

\section{Metodología}

Nuestra iniciativa de innovación parte de los fundamentos del trabajo en equipo, el aprendizaje basado en proyectos y el trabajo cooperativo.

La experiencia de trabajo en el pequeño grupo orientado a la solución del problema es una de las características distintivas del Aprendizaje Basado en Proyectos (ABP). En estas 
actividades grupales los alumnos toman responsabilidades y acciones que son básicas en su proceso formativo (Kidder, 2012), y por ello se considera que esta forma de trabajo representa una alternativa necesaria en la formación de profesionales, especialmente en el caso de técnicos. Un método que además resulta factible para ser utilizado por los profesores, con mayor o menor intensidad, en la mayor parte de las disciplinas.

A la hora de diseñar una actividad de trabajo cooperativo, es importante tener en cuenta los cinco aspectos que harán que funcione bien la actividad: Interdependencia positiva, Exigibilidad individual, Interacción cara a cara, Habilidades interpersonales y de trabajo en grupo y Reflexión del grupo (Peña, 2010).

La propuesta se enmarca en el contexto de tres asignaturas sobre Gestión y Gobierno de Servicios de Tecnologías de la Información repartidas entre dos grados (informática y telecomunicación) y un máster (informática) impartidos en la Escuela Politécnica de Ingeniería de Gijón. Esta temática tiene una fuerte conexión con el mundo empresarial, ya que aborda la estrategia, diseño, construcción, operación y mejora continua de los servicios basados en las tecnologías de la información y las comunicaciones (TIC). El trabajo en grupo planteado en las asignaturas se orienta hacia la gestión de un servicio real e innovador definido por los miembros de cada equipo, de tal modo que los alumnos puedan trabajar en un entorno similar al empresarial.

La metodología a seguir es común para las tres asignaturas implicadas, dedicándose buena parte de las sesiones de prácticas de laboratorio al desarrollo de los trabajos en grupo de la asignatura. Con estas sesiones presenciales, los alumnos disponen de tiempo suficiente para interacción cara a cara, discusión de ideas, toma de decisiones, etc. y permite a los profesores una valoración directa de la comunicación en los equipos, íntimamente relacionada con la cooperación. Además, los alumnos disponen de tiempo no presencial en la planificación de la asignatura para avanzar en el trabajo de forma individual, potenciando la cooperación en este caso mediante las herramientas TIC proporcionadas, por ejemplo, la comunicación vía mensajería instantánea o Chat.

La herramienta TIC utilizada por parte de los equipos de trabajo para mejorar la cooperación entre sus miembros es Teams de Office 365, que ofrece un entorno colaborativo para que los estudiantes puedan interactuar y compartir información relativa a proyectos comunes. Entre las funcionalidades proporcionadas por Teams podemos destacar mensajería instantánea, audioconferencia, videoconferencia, planificación de reuniones y bloc de notas de clase compartido (estructurado en tres partes principales, una de solo lectura para los alumnos, otra colaborativa entre los miembros de equipo y otra privada para cada alumno que solo puede ver el profesor). La herramienta está además disponible tanto para equipos de escritorio como para dispositivos móviles (apps para Android e iOS), de modo que permite la colaboración desde cualquier ubicación. 


\subsection{Plan de trabajo desarrollado}

Las tareas llevadas a cabo para cubrir los objetivos planteados son las siguientes:

Objetivo 1: Familiarizar al alumno con herramientas y estrategias de trabajo cooperativo.

1. Concienciar al alumno sobre la importancia de la cooperación en los desarrollos en equipo en el contexto laboral.

2. Tutorar a los alumnos sobre las estrategias utilizadas en aprendizaje cooperativo.

3. Plantear el uso de las herramientas TIC propuestas como base del trabajo cooperativo de los alumnos.

4. Plantear trabajos en equipo de carácter realista a desarrollar por parte de entre 3 y 4 alumnos durante unas 12 semanas y en los que se garantice tanto la interdependencia positiva como la interacción cara a cara.

Objetivo 2: Medición y monitorización de la cooperación alcanzada.

5. Extraer medidas de las variables de interés que permitan las herramientas de cooperación utilizadas y utilizarlas como métricas.

6. Valorar el grado de cooperación percibido por los profesores durante las sesiones presenciales de trabajo en equipo y utilizarlo como métrica.

Objetivo 3: Estimación del rendimiento de los equipos.

7. Valorar el rendimiento alcanzado por los equipos al final del período de desarrollo del trabajo en equipo en base los resultados alcanzados.

8. Analizar la relación entre el rendimiento alcanzado por los equipos y los valores de las métricas de cooperación seleccionadas.

9. Obtener las expresiones que mejor estimen el rendimiento de los equipos y los miembros de los equipos a partir de las métricas de cooperación seleccionadas.

\section{Resultados}

Se resumen a continuación los resultados obtenidos en relación a cada objetivo planteado.

Objetivo 1: Familiarizar al alumno con herramientas y estrategias de trabajo cooperativo.

En este sentido la experiencia ha sido satisfactoria tanto para docentes como alumnos. Se realizó una encuesta final a los alumnos donde se les pedía valorar la contribución de la herramienta Teams sobre varios aspectos del trabajo en equipo de la asignatura: 
Coordinación, Comunicación, Cooperación, Sentimiento de equipo y Resultados alcanzados. La media global obtenida para las tres asignaturas del estudio fue de 5,23 en una escala de likert de 7 niveles.

Objetivo 2: Medición y monitorización de la cooperación alcanzada.

En la tabla 1 se muestran las métricas cuya obtención fue finalmente viable. Las métricas de comunicación vía Chat se obtuvieron directamente a partir de los informes de uso de la herramienta Teams. Inicialmente se pretendía obtener estas métricas una vez a la semana aproximadamente para analizar la evolución a lo largo del semestre, pero no fue posible debido a que los informes de Teams de momento solo pueden ser generados por parte del administrador general de la herramienta. Así las cosas, solo fue posible y razonable obtener las métricas agregadas al final del semestre.

Tabla 1. Métricas de cooperación

\begin{tabular}{|c|l|l|}
\hline Métrica & \multicolumn{1}{|c|}{ Definición } & \multicolumn{1}{c|}{ Forma de cálculo } \\
\hline Chat & $\begin{array}{l}\text { Comunicación vía } \\
\text { Chat individual }\end{array}$ & $\begin{array}{l}\text { Linealmente entre 1 mensaje y la mitad de la media } \\
\text { para el total de alumnos }\end{array}$ \\
\hline Med.Chat & $\begin{array}{l}\text { Comunicación vía } \\
\text { Chat en el equipo }\end{array}$ & $\begin{array}{l}\text { Media de las métricas individuales de los miembros } \\
\text { del equipo }\end{array}$ \\
\hline Desv.S.Chat & $\begin{array}{l}\text { Desviación de la } \\
\text { comunicación vía } \\
\text { Chat en el equipo }\end{array}$ & $\begin{array}{l}\text { Desviación estándar de las métricas individuales de } \\
\text { los miembros del equipo }\end{array}$ \\
\hline Com & $\begin{array}{l}\text { Comunicación } \\
\text { oral en el equipo }\end{array}$ & $\begin{array}{l}\text { Valoración subjetiva por parte del profesor de la } \\
\text { comunicación oral entre los miembros del equipo } \\
\text { durante las sesiones presenciales de trabajo en } \\
\text { equipo (unas 20 horas en total) }\end{array}$ \\
\hline
\end{tabular}

En la figura 1 se muestran los valores de la métrica Chat a nivel individual para los 73 alumnos correspondientes a las tres asignaturas incluidas en el estudio. Se puede apreciar que en torno al $11 \%$ de los alumnos han intercambiado un número de mensajes con sus compañeros de equipo significativamente mayor que el resto. Además, hay un $4 \%$ de alumnos que no han intercambiado mensajes y optaron por utilizar su propio servicio de mensajería instantánea. Los alumnos de estos dos extremos corresponden casi todos ellos a la asignatura de máster y es por ello que no se ha incluido esta asignatura (15 alumnos en total) en el análisis de la correlación con el rendimiento de los equipos. 


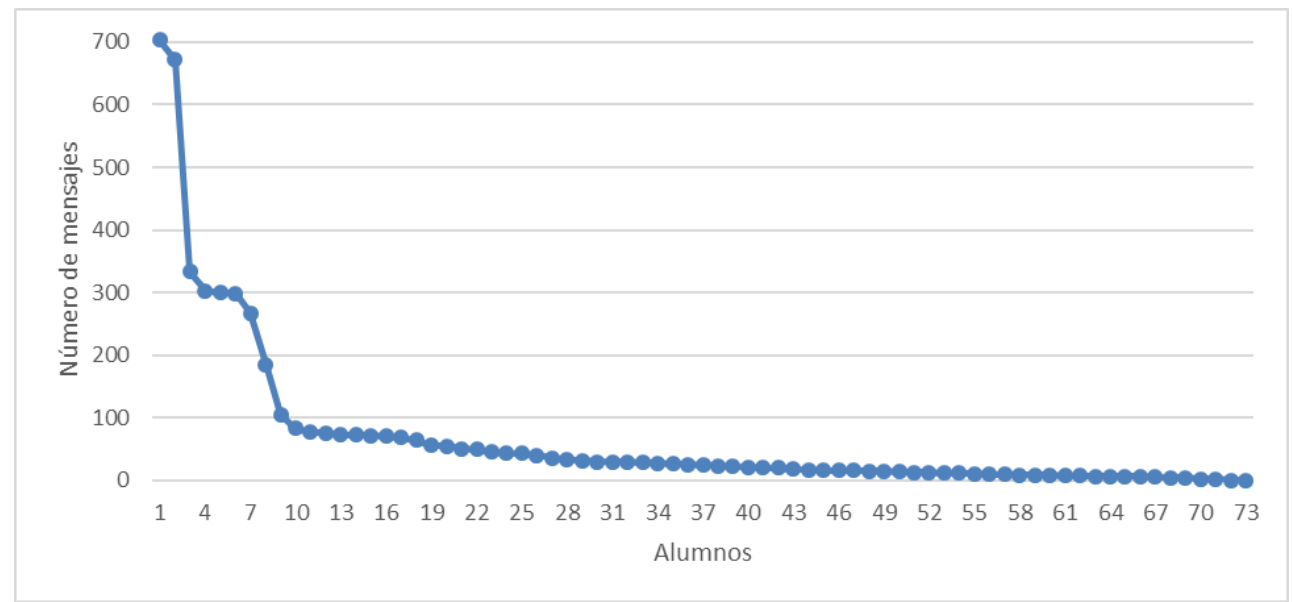

Figura 1. Valores de la métrica Chat a nivel individual

En cuanto a la métrica de comunicación oral solo fue posible su valoración a nivel de equipo por parte de los profesores, quedando pendiente como reto para un futuro la medición del nivel de comunicación oral de cada miembro del equipo. Además, estas métricas solo se han cuantificado para las dos asignaturas del grado, si bien representan el $80 \%$ de los alumnos.

En la figura 2 se muestran los valores de las métricas a nivel de equipo. En el caso de las métricas de Chat se observa una relación entre valores altos de la media y bajos de la desviación estándar y viceversa.

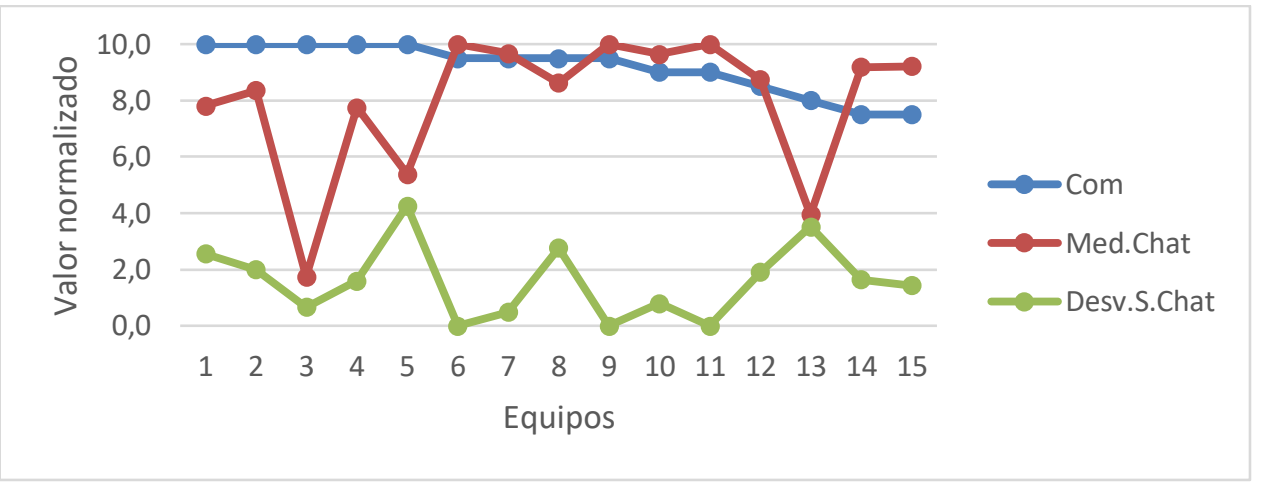

Figura 2. Valores de las métricas a nivel de equipo

Objetivo 3: Estimación del rendimiento de los equipos.

Este objetivo se ha orientado únicamente a las dos asignaturas de grado, con un total de 58 alumnos divididos en 15 grupos. El significativo menor número de alumnos (15) y equipos 
(4) en la asignatura de máster, así como el hecho de no disponer de información sobre el nivel de comunicación oral en los equipos, descartaron finalmente su inclusión.

Una vez concluida la evaluación de las asignaturas y conocida la calificación de los trabajos en equipo, se analizó en profundidad su correlación con las métricas de cooperación obtenidas. La calificación obtenida por los alumnos en el trabajo en equipo consta de dos partes, una común a todos sus miembros y que depende de los resultados alcanzados, y otra individual que depende de la defensa del trabajo realizada por cada uno de ellos.

En primer lugar, y como estrategia prioritaria, se buscó la mejor estimación lineal posible de la parte común de la calificación del trabajo en equipo a partir de las métricas. La expresión encontrada por parte de la herramienta utilizada (Excel) fue la siguiente:

$$
\text { Calificación estimada }=0,484 * \text { Com }-0,013 * \text { Med.Chat }-0,069 * \text { Desv.S.Chat }
$$

donde el valor de los coeficientes asociados a las métricas da idea de su peso relativo.

La estimación anterior da lugar a un error predicción (diferencia entre calificaciones reales y estimadas) de 0,281 (medio) $\pm 0,260$ (desviación estándar) para el conjunto de 15 equipos en las dos asignaturas. Este error es un 6,4\% inferior al obtenido utilizando solo la métrica Com en la predicción y un 33,4\% inferior al obtenido utilizando solo las dos métricas de Chat. En la figura 3 se muestran las calificaciones reales en orden decreciente y las estimaciones correspondientes. El error de estimación es superior a 0,5 en solo 3 de los 15 equipos.

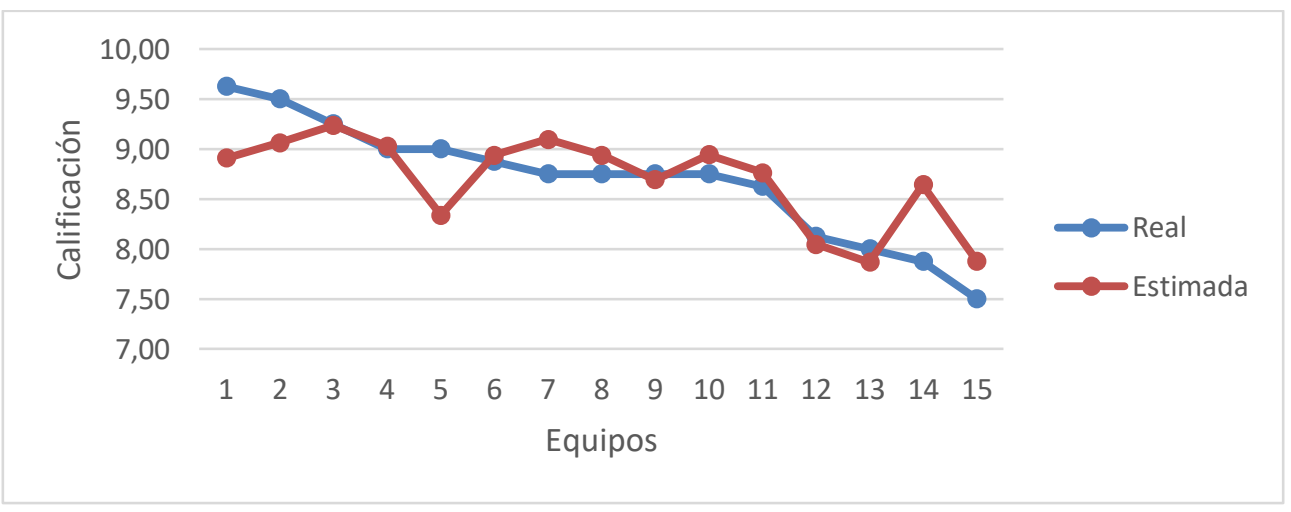

Figura 3. Estimación del rendimiento de los equipos

En segundo lugar, y aunque no planteado inicialmente en el trabajo, se buscó la mejor estimación lineal posible de la parte individual de la calificación del trabajo en equipo. La expresión encontrada fue la siguiente:

Calificación estimada $=0,388 *$ Com $-0,025 *$ Med.Chat $-0,040 *$ Desv.S.Chat 
donde el valor de los coeficientes asociados a las métricas da idea de su peso relativo.

La estimación anterior da lugar a un error predicción de 0,283 \pm 0,234 para el conjunto de 58 alumnos en las dos asignaturas. Este error es un 5\% inferior al obtenido utilizando solo la métrica Com en la predicción y un 30,4\% inferior al obtenido utilizando solo las dos métricas de Chat. En la figura 4 se muestran las calificaciones reales en orden de equipos y las estimaciones correspondientes. El error de estimación es superior a 0,5 en solo en 9 alumnos $(15,5 \%)$ pertenecientes a los mismos 3 equipos mencionados anteriormente.

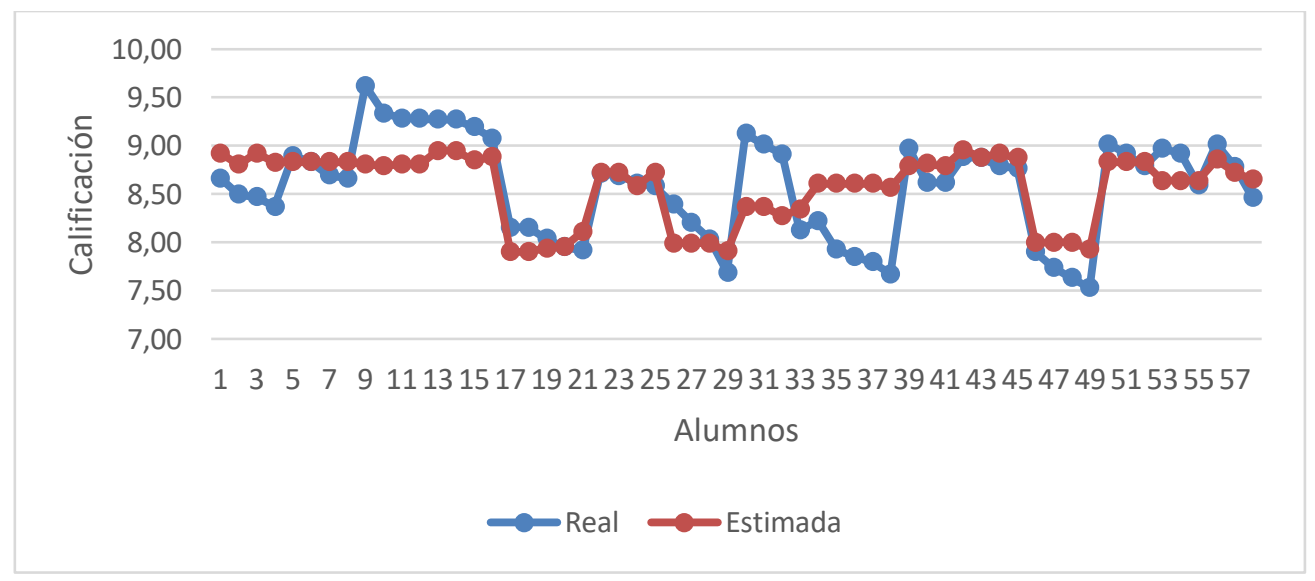

Figura 4. Estimación del rendimiento de los miembros de los equipos

\section{Conclusiones}

Como puntos fuertes del trabajo realizado destacamos la buena valoración de la herramienta de trabajo cooperativo, la definición de varias métricas para valorar la cooperación de equipos y miembros y la obtención de estimaciones del rendimiento tanto de equipos como de sus miembros a partir de las métricas de cooperación definidas. La estimación del rendimiento de equipos y alumnos habilita la monitorización en tiempo real de la cooperación, que podría ser útil a profesores y alumnos para la detección temprana de problemas de funcionamiento en los equipos. Con ese objetivo, se plantean las métricas relativas a comunicación oral y vía Chat frente a otras como las relativas a foros, wikis y repositorios de código.

Como aspecto vital de mejora, la monitorización en tiempo real de la cooperación debe pasar necesariamente por disponer de valores periódicos de las métricas. Disponer de datos de comunicación vía Chat de forma periódica (p.e. semanalmente) supondría conocer no solo el nivel sino también el patrón temporal de comunicación, lo que permitiría componer 
métricas adicionales (p.e. a partir de la varianza) que aportarían sin duda mayor precisión a las estimaciones de rendimiento. Esperamos que una próxima actualización de la herramienta Teams permita resolver a corto plazo la limitación actual. En cuanto a la medición de la comunicación oral, automatizar dicha medición mediante micrófonos asociados a los alumnos en las sesiones presenciales de trabajo en equipo permitiría dar un gran salto de calidad en esta importante métrica. No solo la precisión de las medidas sería notablemente superior, sino que además se abriría la posibilidad de componer nuevas métricas que reflejen el patrón temporal de comunicación, al igual que ocurría con la comunicación vía Chat.

\section{Referencias}

Anaya, A.R. and Boticario, J.G. (2011). Application of machine learning techniques to analyse student interactions and improve the collaboration process. Expert Systems with Applications, Elsevier, 38.

Chawinga, W.D. (2017). Taking social media to a university classroom: teaching and learning using Twitter and blogs. Int. Journal of Educational Technology in Higher Education, 14(3).

INTEF (2017). Resumen Informe Horizon, Educación Superior. Instituto Nacional de Tecnologías Educativas y de Formación de Profesorado (INTEF).

Kidder, D. and Bowes-Sperry, L. (2012). Examining the influence of team project design decisions on student perceptions and evaluations of instructors. Academy of Mgmt. Learning \& Education, 11 (1).

Peña, J.D. (2010). El aprendizaje cooperativo y las competencias. Revista d'Innovació Docent Universitària (RIDU), 2.

Perera, D. et Al. (2009). Clustering and Sequential Pattern Mining of Online Collaborative Learning Data. IEEE Transactions on Knowledge and Data Engineering, 21(6).

Viswanathan, S. A. and VanLehn, K. (2017). Using the tablet gestures and speech of pairs of students to classify their collaboration. IEEE Transactions on Learning Technologies, 11(2), 230-242. 